\title{
Consequences of CT colonography in stenosing colorectal cancer
}

\author{
JF Huisman ${ }^{1} \cdot$ LW Leicher ${ }^{1} \cdot$ E de Boer ${ }^{2} \cdot$ HL van Westreenen ${ }^{3} \cdot$ JW de Groot ${ }^{4}$. \\ FA Holman ${ }^{5} \cdot$ PC van de Meeberg ${ }^{6}$. PEJM Sallevelt ${ }^{7}$ - KCMJ Peeters ${ }^{5}$. \\ MNJM Wasser $^{8} \cdot$ HFA Vasen $^{9} \cdot$ WH de Vos tot Nederveen Cappel $^{1}$
}

Accepted: 11 October 2016/Published online: 25 October 2016

(C) Springer-Verlag Berlin Heidelberg 2016

\begin{abstract}
Background In patients with stenosing colorectal cancer (CRC), visualization of the entire colon prior to surgery is recommended to exclude synchronous tumors. Therefore, most centers combine computed tomographic colonography (CTC) with staging CT. The aims of this study were to evaluate the yield and clinical implications of CTC.

Methods In this multicenter retrospective study, patients with stenosing CRC that underwent CTC and subsequent surgery between April 2013 and November 2015 were included. Result of the CTC, its influence on the surgical treatment plan, and final histology report were evaluated.
\end{abstract}

Huisman JF and Leicher LW equally contributed to this manuscript.

JF Huisman

j.f.huisman@isala.nl

1 Department of Gastroenterology and Hepatology, P.O. box 10400, Isala, 8000 GK Zwolle, the Netherlands

2 Department of Radiology, Isala, Zwolle, the Netherlands

3 Department of Surgery, Isala, Zwolle, the Netherlands

4 Department of Medical Oncology, Isala, Zwolle, the Netherlands

5 Department of Surgery, Leiden University Medical Center, Leiden, the Netherlands

6 Department of Gastroenterology and Hepatology, Slingeland hospital, Doetinchem, the Netherlands

7 Department of Radiology, Slingeland hospital, Doetinchem, the Netherlands

8 Department of Radiology, Leiden University Medical Center, Leiden, the Netherlands

9 Dept of Gastroenterology and Hepatology, Leiden University Medical Center, Leiden, the Netherlands
Results One hundred sixty-two patients with stenosing CRC were included. Nine $(5.6 \%)$ synchronous cancers proximal to the stenosing tumor were suspected with CTC. In four of nine patients, the CTC did not change the primary surgical plan because the tumors were located in the same surgical segment. In five of nine patients, CTC changed the surgical treatment plan. Three of these five patients underwent an extended resection and the presence of the tumors was confirmed. Two of these three synchronous CRCs were also visible on abdominal staging CT. In the other two patients, the result of CTC was false positive which led to an unnecessary extended resection in one patient.

Conclusion The yield of CTC was relatively low. In only three patients $(1.9 \%)$, CTC correctly changed the primary surgical plan, but in two of them, the tumor was also visible on abdominal staging CT. Moreover, in two patients, CTC was false positive. The clinical value of CTC in stenosing CRC appears to be limited.

Keywords Colorectal cancer · CT colonography · Stenosing · Obstructive

Synopsis In stenosing CRC, computed tomographic colonography (CTC) is performed next to thoracic and abdominal staging CT to exclude synchronous CRC. This study demonstrates limited added value of CTC and also possible harm due to false positive results.

\section{Introduction}

Colorectal cancer (CRC) is the second most common cause of cancer related death in the Western world [1]. In 2012, 471.000 new cases were diagnosed in Europe and 134.000 in the USA [1]. In more than half of the cases, the tumor is 
located in the left part of the colon [2]. At the time of presentation, $45 \%$ of symptomatic patients have metastatic disease [3].

Of all patients with CRC, 15-20\% present with stenosing CRC. In these patients, colonoscopy might fail to diagnose synchronous tumors proximal to the stenosing cancer which may result in secondary surgery [4-8]. A synchronous tumor is reported in 1-7\% of the patients with CRC [9-11]. In two thirds of the cases, both tumors are located in the same surgical segment $[10,12]$.

Computed tomographic colonography (CTC) is developed as a non-invasive tool for the detection of CRC and polyps as an alternative to colonoscopy. CTC is highly sensitive (96\%) in the screening for CRC [13-15]. In patients with stenosing CRC, Park et al. demonstrated a sensitivity of $100 \%$ of CTC in the detection of proximal synchronous CRC and moderate sensitivity $(88.6 \%)$ in detecting proximal synchronous adenomas, including advanced adenomas. Specificity was 69.8 and $78.8 \%$ for the detection of CRC and adenomas, respectively [16].

In patients with stenosing $\mathrm{CRC}, \mathrm{CTC}$ is recommended by most authorities to exclude synchronous CRC [17-20]. Two previous studies described a change in primary surgical plan because of CTC in respectively 14 and $16 \%$ of patients with stenosing CRC due to location errors, synchronous adenomas, or synchronous carcinomas [21, 22]. However, in most cases of stenosing CRC, the tumor is in T-stage 3 or 4 and therefore visible on regular staging $\mathrm{CT}$, that is nowadays performed in all patients with CRC prior to surgery. Furthermore, improved endoscopic techniques may prevent patients from unnecessary performed surgery because of (advanced) synchronous adenomas or early carcinomas.

The aims of our study were to evaluate the yield and added clinical implications of CTC in patients with stenosing CRC.

\section{Materials and methods}

This multicenter retrospective observational cohort study was performed in three Dutch hospitals: Isala in Zwolle, Leiden University Medical Center (LUMC) in Leiden and Slingeland hospital in Doetinchem. Patients were included between 1 April 2013 and 1 November 2015. The study was approved by the institutional ethical committees.

\section{Patients}

In this study, stenosing CRC is defined as colorectal cancer diagnosed with colonoscopy and not able to pass by the endoscopist due to stenosing of the lumen by the tumor. Subsequently, the colon proximal to the tumor is not inspected. Obstructive CRC is defined as colorectal cancer presenting with symptoms requiring emergency surgery or stent placement. Preoperative endoscopy with adequate inspection of the colon mucosa in these patients is not possible.

All patients with CRC were discussed in the multidisciplinary CRC team. Patients that underwent incomplete colonoscopy due to stenosing CRC followed by preoperative CTC and subsequent surgical resection were included. Symptomatic patients that presented with obstructive CRC and subsequently underwent emergency surgery without preoperative colonoscopy and CTC and patients that did not undergo surgical resection because of advanced disease were excluded. Figure 1 presents a flowchart of included and excluded patients. Data on sex, age, tumor location, cancer stage, result on abdominal CT, outcome of CTC, and type of surgery as well as data on the postoperative colonoscopy were collected. A change in primary surgical plan was defined as a surgical procedure other then would be performed for stenosing CRC only.

\section{Preoperative imaging}

Most patients who complied with the inclusion criteria underwent colonoscopy and a combined CTC with abdominal and thoracic staging CT. In some patients (i.e., patients with abdominal pain), an abdominal CT had already been performed prior to colonoscopy. In these patients, additional CTC and thoracic staging CT were performed. Tumor location with colonoscopy and CTC (i.e., rectum, sigmoid, descending colon, splenic flexure, transverse colon, hepatic flexure, ascending colon, and caecum) was documented. All CT images were analyzed by experienced radiologists who had more than 400 CTC case experiences.

\section{CTC technique}

CTC examinations were performed using Philips Ingenuity $\mathrm{CT}$ in Isala, Siemens Somatom in Slingeland and Toshiba Aqcuilion One in LUMC (Table 1).

Participants received bowel preparation consisting of $3 \times 50 \mathrm{~mL}$ of iodinated contrast agent (Telebrix Gastro) on the day prior to CTC combined with a low fiber diet for 1 day. Immediately before CT scanning, $2 \mathrm{~mL}$ scopolaminebutyl $(20 \mathrm{mg} / \mathrm{mL})$ was injected intravenously and colon distension was achieved with an automatic $\mathrm{CO}_{2}$ insufflator using a rectal catheter. CTC images were obtained with the patient in prone and supine position. Abdominal and thoracic staging was performed during portal venous phase and during arterial phase after intravenously administering of iodinated contrast. CTC software reconstructed 2-dimensional (2D) and 3-dimensional (3D) images of the bowel. In Isala and Slingeland hospital, 2D and $3 \mathrm{D}$ reading strategy were used, in LUMC 2D, strategy only.

CTC computed-aided diagnosis (CAD) system was used as an automatic warning system for bowel wall abnormalities. 
Fig. 1 A flowchart of included and excluded patients

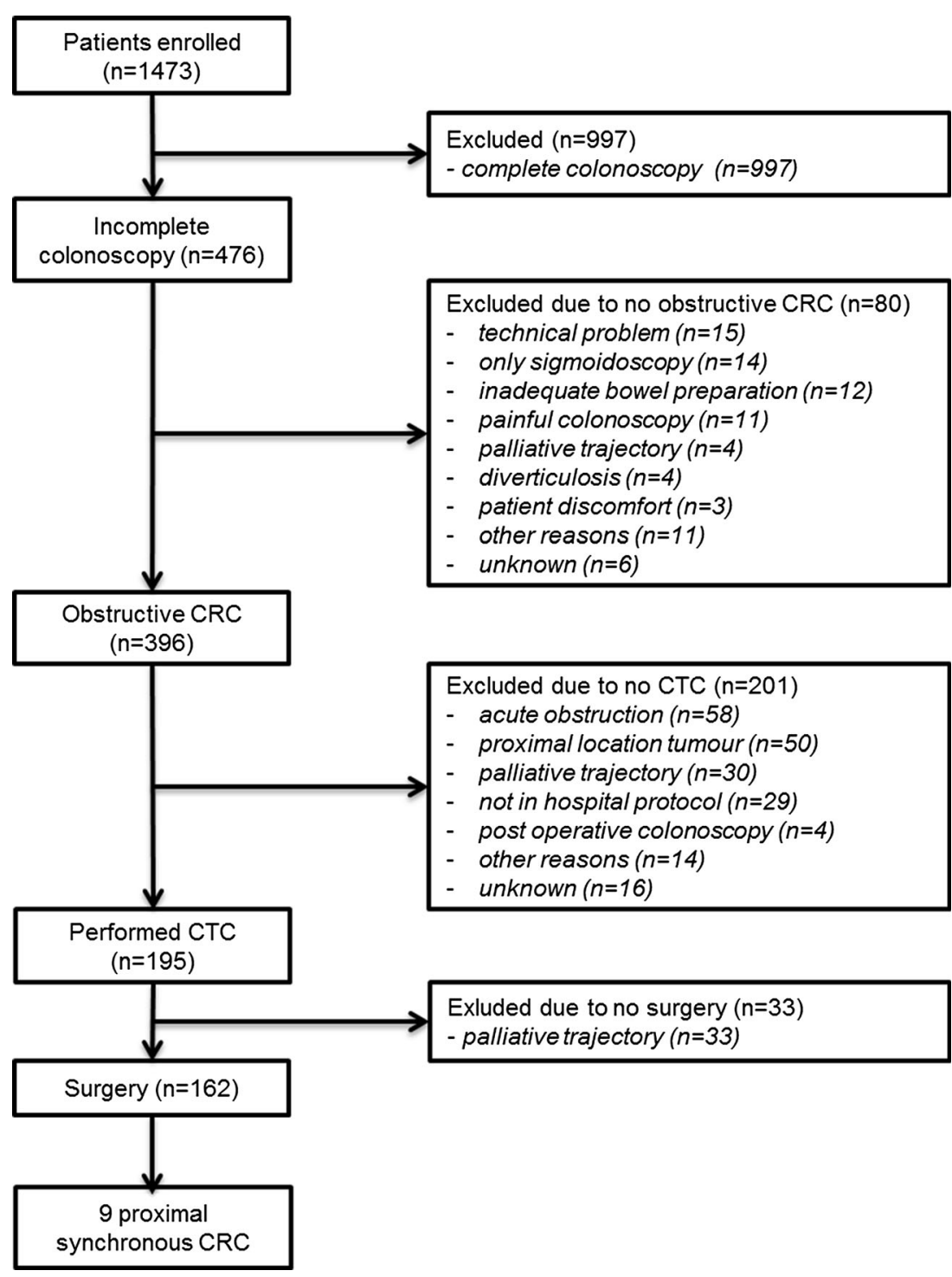

\section{Statistics}

Descriptive statistics were performed using Statistical Package of Social Sciences version 23 (SPSS). True positives were defined as tumors detected by CTC and confirmed by surgery and pathological examination. False positives were tumors detected by CTC, but not confirmed by surgery or follow-up.

\section{Results}

\section{Patient characteristics}

In the multidisciplinary team, 1473 patients with CRC were discussed. One thousand three hundred eleven patients (89\%) were excluded because of various reasons complete preoperative colonoscopy performed $(n=997)$, incomplete colonoscopy not due to stenosing CRC $(n=80)$, emergency surgery necessary $(n=58)$, preoperative CTC not performed $(n=143)$, no surgical resection performed due to advanced disease ( $n=33$ ) (Fig. 1). A total of 162 patients (male $n=85$, $52.4 \%$ ) with a median age of $71 \pm 10$ years complied the inclusion criteria.

\section{CTC quality}

No complications of CTC were described. In two cases, CTC did not succeed due to poor bowel distension. In the remaining 160 patients, in $131 / 160$ patients ( $80.9 \%$ ) CTC could be assessed reliable as reported by the radiologist. In 29 patients, CTC quality was poor due to inadequate bowel distension $(n=21)$, large amount of weakly tagged fecal matter $(n=6)$ or an unknown reason $(n=2)$. 
Table 1 CTC protocol Isala, LUMC, and Slingeland hospital

\begin{tabular}{|c|c|c|c|}
\hline & Isala & LUMC & Slingeland \\
\hline Type CT scan & $\begin{array}{l}\text { Philips ingenuity } \\
\text { CT } 256 \text { slices }\end{array}$ & $\begin{array}{r}\text { Toshiba aqcuilion } \\
\text { one (320 slice) }\end{array}$ & $\begin{array}{l}\text { Siemens somatom definition } \\
\text { AS 64-slice configuration }\end{array}$ \\
\hline \multicolumn{4}{|l|}{ Scan parameters } \\
\hline - Collimation (mm) & $128 \times 0.625$ & $320 \times 0.5$ & $64 \times 0.6$ \\
\hline - Beam pitch & 0.899 & - & 0.9 \\
\hline - Rotation time (sec) & 0.75 & 0.5 & 0.5 \\
\hline - Slice thickness (mm) & 0.9 & 1 & - \\
\hline - Tube voltage (Kv) & 100 & 120 & 120 \\
\hline - mAs with z modulation & 85 & - & 55 \\
\hline Scan delay (sec) & 70 & 50 & 58 \\
\hline Iodinated contrast & Optiray 350 & Ultravist 370 & Iomeron 300 \\
\hline - Total amount (ml) & 125 & $90-170^{\mathrm{a}}$ & $105-150^{\mathrm{a}}$ \\
\hline - Rate (mL/s) & 4 & $2.4-4.4^{\mathrm{a}}$ & $2-3.9^{\mathrm{a}}$ \\
\hline
\end{tabular}

CT computed tomography, LUMC Leiden University Medical Center

${ }^{\text {a }}$ Depends on body weight

\section{Synchronous CRC}

In nine patients $(5.7 \%)$, a proximal synchronous CRC was suspected on CTC. In three patients, abdominal CT was performed before CTC. In these three cases, the synchronous tumor was already visible on abdominal CT. The time interval between abdominal CT and CTC ranged from 5 to 14 days.

Table 2 provides detailed information about age, sex, tumor location, tumor stage, outcome of $\mathrm{CT}$, change in primary surgical plan, type of surgery, CTC outcome, and time between abdominal CT and CTC of the nine synchronous tumors.

In four of nine patients with synchronous tumors on CTC, the findings of CTC did not change the primary surgical plan. In one of them, the synchronous tumor was already described on the previously performed staging CT scan. In the other three patients, the tumor was located within the scheduled resection (i.e., a right-sided (extended) hemicolectomy in all of them) (Table 2, patients 6-9). Histological examination confirmed synchronous CRC in three of four patients; in the fourth patient (Table 2, patient no. 7), a 35-mm tubulovillous adenoma was diagnosed in the proximal colon.

In five of nine patients with synchronous tumors on CTC, the CTC changed the surgical treatment plan. In three of these five patients, an extended resection was performed and definitive histology showed three synchronous adenocarcinomas (Table 2, patients 3-5). Two of these were $\mathrm{T} 3$ tumors that were also visible on abdominal $\mathrm{CT}$; the third was a T2 tumor and in this patient, a combined CTC with abdominal and thoracic staging was performed. In the other two of five patients (Table 2, patients 1 and 2), the result of CTC was false positive and consequently an unnecessary extended resection was performed in one patient (Fig. 2a, b). In the other patient, only one tumor was detected during surgery. In this patient, a stenosing sigmoid tumor was described with colonoscopy. CTC suspected a synchronous CRC in the ascending colon. However, during surgery, no tumor was palpable in the sigmoid and endoscopic ink patterns were not found in the sigmoid, but in the ascending colon, the suspected sigmoid tumor with colonoscopy was actually located in the ascending colon. Subsequently, the surgeon decided to perform a right-sided hemicolectomy only. In this patient, the false positive result of the CTC led to an open procedure instead of a laparoscopic procedure (Fig. 2c). Postoperative surveillance colonoscopy in this case showed no abnormalities.

\section{Postoperative colonoscopy}

To date, 49 of 162 (30.2\%) patients have undergone postoperative surveillance colonoscopy. The interval between surgery and postoperative colonoscopy varied from 25 days to 2 years, and the mean interval was 8.3 months. No metachronous $\mathrm{CRC}$ was detected at first surveillance colonoscopy.

\section{Discussion}

Most current guidelines recommend preoperative CTC in patients with stenosing CRC [17-20]. Our multicenter retrospective study evaluated the added clinical value of this recommendation. We demonstrated the clinical value of CTC to be very limited. In 3 out of 162 patients, CTC was meaningful in terms of detection of a second primary 


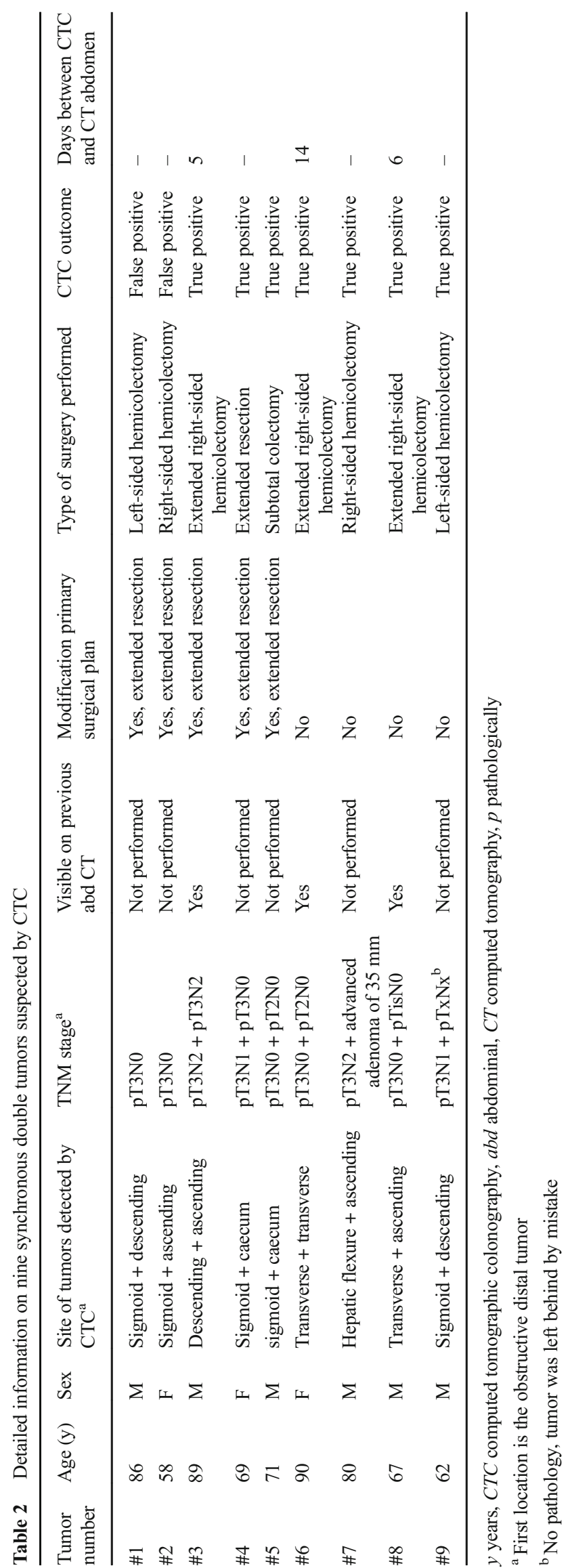

CRC that changed the primary surgical treatment strategy. However, two of these tumors were also detected on the abdominal CT leaving an "added value" in only 1 out of $162(0.6 \%)$ patients with stenosing CRC. Moreover, in two patients, the CTC was false positive leading to an unnecessary extended resection in one patient.

Previous studies reported stenosing CRC in 15-20\% of the cases and synchronous tumors in 1-7 \% [4-11]. CT colonography has similar sensitivity as colonoscopy in detecting CRC and has moderate sensitivity in detecting advanced adenomas [13-15]. Park et al. demonstrated a high sensitivity of CTC for detection of proximal synchronous tumors, but limited capability of CTC in differentiating advanced adenomas from CRC in patients with stenosing CRC [16].

Preoperative CTC has some advantages when compared to colonoscopy performed 3 months after primary surgery: (1) CTC could prevent the need of secondary surgery in case of a synchronous tumor and (2) it could prevent growing of secondary tumors into a more advanced stage when detection and treatment are delayed. But CTC has also some disadvantages: (1) it is another burden for patients, (2) synchronous tumors are often already visible on regular staging CT, (3) sensitivity of CTC is lower in stenosing CRC due to technical difficulties associated with stenosing CRC, and finally, (4) the technique is not able to differentiate between large adenomas and CRC and between $\mathrm{T} 1$ and $\mathrm{T} 2$ tumors that could result in unnecessarily performed extended resections in some patients that could have been treated endoscopically [16, 23].

In three cases $(1.8 \%)$, the scheduled type of surgery had been changed and a more extended surgery was performed. However, in two of these cases, previous performed abdominal CT already showed the second tumor. Two previous studies described a change in surgical plan in 14-16\%, due to location errors, synchronous CRC, or synchronous adenomas $[21,22]$. In these studies, the primary surgical plan was changed in 4 and $11 \%$ due to location errors. However, tattooing colorectal tumors during endoscopy is currently standard of care, which limits the role of CT scan in determination of the location anyway. Moreover, most stenosing tumors are at stage T3 or T4 (for instance in our study in $90 \%$ of the patients) and might therefore likely have been visible on abdominal staging CT, which is performed nowadays in all patients prior to surgery. The presence of a previous performed abdominal CT was not mentioned in these studies. CT colonography can be useful in detecting synchronous $\mathrm{CRC}$ and synchronous adenomas. In the abovementioned studies, the detection of synchronous CRC or adenomas changed the surgical plan in $10(7.3 \%)$ and $5(4.1 \%)$ patients, respectively. Obviously, most adenomas can be 
Fig. 2 CTC images of both false positive CTCs. Red arrows indicate the suspected tumors on CTC. a 3D image of patient number 1, tumor in sigmoid and false tumor in descending colon. $b$ 2D image of patient number 1 , false tumor in descending colon. c 2D image of patient number 2 , false tumor in sigmoid

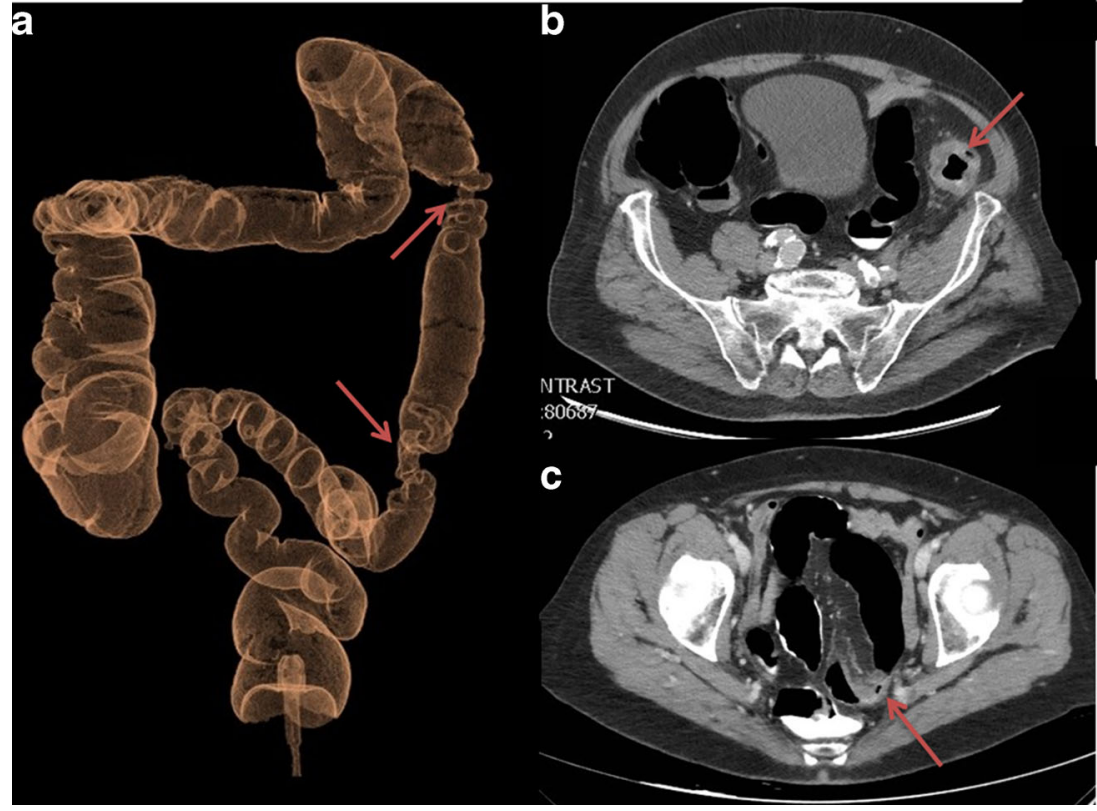

removed endoscopically but also early (T1) carcinomas could be attempted to be removed endoscopically first. The stage of the synchronous tumors was not mentioned in above described studies. In our study, in one of the four patients with suspected synchronous CRC but no change in the primary surgical treatment plan, the postoperative histology showed no synchronous CRC but a proximal 35-mm tubulovillous adenoma.

Another possible disadvantage of CTC is the consequence of a false positive result. In this study, CTC was false positive in two patients $(1.2 \%)$ and the second primary tumor detected by CTC was not confirmed during surgery and at histological examination. This resulted in an unnecessary extended resection in one patient. In the other patient, no tumor was manifested during surgery. In both false positive CTCs, only 2D images were evaluated and suspected for a synchronous CRC at initial diagnosis (Fig. 2). In retrospect, reassessment of these CTCs in 2D by the radiologist, the result of CTC was similar as at initial diagnosis; however, endoluminal 3D images were not suspect for a second tumor and also the CAD system had not warned for an abnormality.

Our study has some limitations. First of all, it has a retrospective design. Secondly, the number of synchronous CRC was relatively low, although the numbers are larger than reported in previous studies. Thirdly, not all surveillance reports were available because they were performed in other surrounding hospitals. Therefore, it cannot be ruled out that postoperative surveillance endoscopies did reveal CRC where CTC was (false) negative. Finally, in Isala and Slingeland hospital, both 2D and 3D reading strategy were used. Some radiologists viewed only 2D images, some used both strategies. In LUMC, only $2 \mathrm{D}$ reading strategy was used. Although a large study showed no significant difference between 2D and 3D reading strategy, CTCs might be false positive using 2D reading strategy only as shown in our study [24].

In conclusion, CTC is highly sensitive in detecting proximal synchronous tumors in patients with stenosing CRC according to previous studies. However, our data suggest very limited clinical benefit of CTC in patients with stenosing CRC and also potential harm in terms of unnecessary extended surgery. In view of our results, a colonoscopy performed, for instance at an interval of 3 months after curative surgery, appears to be a good alternative if full attention is paid to detect synchronous cancers on staging CT. Future prospective studies should be performed to address the question which strategy is the most optimal for patients with stenosing CRC.

Authors' contributions Huisman JF, Leicher LW, and de Vos tot Nederveen Cappel WH were responsible for the study concept and design. Huisman JF collected the data and performed the analyses. Huisman JF, Leicher LW, de Vos tot Nederveen Cappel WH, and Vasen HFA interpreted the data. Huisman JF and Leicher LW drafted the manuscript. All authors have critically revised the manuscript for its intellectual contents.

Compliance with ethical standards This retrospective study was approved by the institutional ethical committees.

Disclosure of interest None declared. 
Financial conflicts This study was not funded.

\section{References}

1. Ferlay J, Soerjomataram I, Dikshit R et al (2015) Cancer incidence and mortality worldwide: sources, methods and major patterns in GLOBOCAN 2012. Int J Cancer 136(5):E359-E386

2. Siegel R, Desantis C, Jemal A (2014) Colorectal cancer statistics, 2014. CA Cancer J Clin 64(2):104-117

3. Moreno CC, Mittal PK, Sullivan PS, et al. (2015) Colorectal cancer initial diagnosis: screening colonoscopy, diagnostic colonoscopy, or emergent surgery, and tumor stage and size at initial presentation. Clin Colorectal Cancer

4. Billingsley KG, Morris AM, Dominitz JA et al (2007) Surgeon and hospital characteristics as predictors of major adverse outcomes following colon cancer surgery: understanding the volumeoutcome relationship. Arch Surg 142(1):23-31 discussion 32

5. Deans GT, Krukowski ZH, Irwin ST (1994) Malignant obstruction of the left colon. Br J Surg. 81(9):1270-1276

6. Serpell JW, McDermott FT, Katrivessis H, Hughes ES (1989) Obstructing carcinomas of the colon. Br J Surg. 76(9):965-969

7. Ascanelli S, Navarra G, Tonini G et al (2003) Early and late outcome after surgery for colorectal cancer: elective versus emergency surgery. Tumori 89(1):36-41

8. Ohman U (1982) Prognosis in patients with obstructing colorectal carcinoma. Am J Surg 143(6):742-747

9. Lam AK, Chan SS, Leung M (2014) Synchronous colorectal cancer: clinical, pathological and molecular implications. World J Gastroenterol 20(22):6815-6820

10. Mulder SA, Kranse R, Damhuis RA et al (2011) Prevalence and prognosis of synchronous colorectal cancer: a dutch populationbased study. Cancer Epidemiol 35(5):442-447

11. Adloff M, Arnaud JP, Bergamaschi R, Schloegel M (1989) Synchronous carcinoma of the colon and rectum: prognostic and therapeutic implications. Am J Surg 157(3):299-302

12. Latournerie M, Jooste V, Cottet V, Lepage C, Faivre J, Bouvier AM (2008) Epidemiology and prognosis of synchronous colorectal cancers. Br J Surg 95(12):1528-1533
13. Pickhardt PJ, Hassan C, Halligan S, Marmo R (2011) Colorectal cancer: CT colonography and colonoscopy for detection - systematic review and meta-analysis. Radiology 259(2):393-405

14. Halligan S, Altman DG, Taylor SA et al (2005) CT colonography in the detection of colorectal polyps and cancer: systematic review, meta-analysis, and proposed minimum data set for study level reporting. Radiology 237(3):893-904

15. Mulhall BP, Veerappan GR, Jackson JL (2005) Meta-analysis: computed tomographic colonography. Ann Intern Med 142(8):635-650

16. Park SH, Lee JH, Lee SS et al (2012) CT colonography for detection and characterisation of synchronous proximal colonic lesions in patients with stenosing colorectal cancer. Gut 61(12):1716-1722

17. AGA Clinical Practice and Economics Committee (2006) Position of the american gastroenterological association (AGA) institute on computed tomographic colonography. Gastroenterology 131(5): $1627-1628$

18. Hong N, Park SH (2014) CT colonography in the diagnosis and management of colorectal cancer: emphasis on pre- and postsurgical evaluation. World J Gastroenterol 20(8):2014-2022

19. Cash BD, Rockey DC, Brill JV (2011) AGA standards for gastroenterologists for performing and interpreting diagnostic computed tomography colonography: 2011 update. Gastroenterology 141(6): 2240-2266

20. Spada C, Stoker J, Alarcon O et al (2014) Clinical indications for computed tomographic colonography: European society of gastrointestinal endoscopy (ESGE) and european society of gastrointestinal and abdominal radiology (ESGAR) guideline. Endoscopy 46(10):897-915

21. Flor N, Ceretti AP, Mezzanzanica M et al (2013) Impact of contrastenhanced computed tomography colonography on laparoscopic surgical planning of colorectal cancer. Abdom Imaging 38(5): 1024-1032

22. Kim JH, Kim WH, Kim TI et al (2007) Incomplete colonoscopy in patients with occlusive colorectal cancer: usefulness of CT colonography according to tumor location. Yonsei Med J 48(6): 934-941

23. Sali L, Falchini M, Taddei A, Mascalchi M (2014) Role of preoperative CT colonography in patients with colorectal cancer. World J Gastroenterol 20(14):3795-3803

24. Johnson CD, Chen M, Toledano AY et al (2008) Accuracy of CT colonography for detection of large adenomas and cancers. N Engl J Med 359(12):1207-1217 